\title{
Medida do Grau de Relacionamento entre Estímulos Equivalentes
}

\author{
Measuring the Relatedness of Equivalent Stimuli \\ Renato Bortolotia \& Júlio C. de Rose ${ }^{* b}$ \\ ${ }^{a}$ Universidade Federal do Pará, Belém, Brasil \\ ${ }^{\mathrm{b}}$ Universidade Federal de São Carlos, São Carlos, Brasil
}

\begin{abstract}
Resumo
Este estudo apresenta um procedimento para verificar a existência de "graus de relacionamento" diferentes entre estímulos equivalentes por meio de uma medida quantitativa de compartilhamento de funções. Dois grupos de estudantes universitários participaram do estudo. Os participantes do grupo experimental estabeleceram classes de equivalência entre figuras abstratas e fotografias de faces expressando alegria, raiva e nojo. Em seguida, avaliaram algumas das figuras abstratas usando um instrumento de diferencial semântico. O mesmo instrumento foi utilizado na avaliação das faces e figuras pelos participantes do grupo controle, não treinados a estabelecer relações entre esses estímulos. Esses últimos participantes avaliaram as figuras como neutras e as avaliações que fizeram das faces corresponderam às avaliações das figuras equivalentes a elas feitas pelos participantes do grupo experimental. A comparação entre os valores atribuídos às faces e às figuras dá uma medida quantitativa do grau de relacionamento entre esses estímulos e essa medida pode ser usada no estudo de parâmetros da formação de classes como o número de nódulos.

Palavras-chave: Equivalência de estímulos; transferência de funções; medida do significado; estudantes universitários.

Abstract

There are methodological difficulties to quantify the relatedness of equivalent stimuli. The purpose of this study was to create an instrument that could be helpful in this process. Two groups of college students took part in this study. The experimental group established equivalence classes comprised of abstract pictures and pictures of faces expressing anger, happiness and disgust. They then evaluated some of these pictures with a semantic differential. The control group used the same instrument in evaluating faces and figures, untrained in establishing relations between those stimuli. The control group assessed the figures as neutral and their assessment of the faces corresponded to that carried out by the participants of the experimental group - to them, the figures were equivalents to the faces. The comparison between the values attributed to the faces and figures provides a quantitative measurement of the degree of relatedness between those stimuli and that may be used to study parameters such as the number of nodes.

Keywords: Stimuli equivalence; transfer of functions; assessment of meaning; college students.
\end{abstract}

A linguagem e outras manifestações simbólicas que têm seu significado compartilhado por um grupo social podem ser compreendidas como sistemas artificiais que adquirem suas características em função do papel que exercem na vida desse grupo. Mais especificamente, os símbolos são relacionados aos seus referentes por meio de convenções sociais arbitrárias que são mantidas por práticas culturais e lingüísticas presentes nos meios sociais em que elas se

\footnotetext{
* Endereço para correspondência: Departamento de Psicologia, Universidade Federal de São Carlos, Caixa Postal 676, São Carlos, SP, 13565-905. Fone/fax: (16) 3351-8492. E-mail: djcc@power.ufscar.br

Baseado em dissertação apresentada pelo primeiro autor como parte dos requisitos para a obtenção do mestrado em Teoria e Pesquisa do Comportamento, na Universidade Federal do Pará. Trabalho realizado com apoio do PRONEX/ CNPq. A preparação do manuscrito contou com apoio da FAPESP, Processo 03/09928-4. Agradecemos à contribuição de Olavo de Faria Galvão para este trabalho.
}

desenvolveram. Nessas convenções, símbolos e referentes, elementos muitas vezes completamente distintos, são relacionados de modo que um possa substituir o outro em muitos contextos, ou seja, eles se tornam equivalentes em uma ampla variedade de circunstâncias (Sidman, 1994).

O modelo da equivalência de estímulos proposto por Sidman e Tailby (1982) estabeleceu critérios operacionais que permitiram identificar relações simbólicas e simular a aquisição delas em laboratório. Os estudos experimentais que adotam o modelo de equivalência de estímulos normalmente utilizam procedimentos de emparelhamento ao modelo. Por meio desses procedimentos, são estabelecidas relações condicionais entre cada estímulo de um conjunto de modelos e um estímulo correspondente de um conjunto de comparações: o participante deve escolher um entre dois ou mais estímulos de comparação condicionalmente à apresen- 
tação de um estímulo modelo. Por convenção, denomina-se $\mathrm{AB}$ a relação entre os estímulos modelo do conjunto A e os estímulos de comparação do conjunto B. Cada conjunto de estímulos tem dois ou mais membros, representados por combinações alfanuméricas. No caso da relação AB, na presença do modelo A1, a escolha do estímulo de comparação B1 é indicada como correta, na presença do modelo A2, a escolha do estímulo de comparação B2 é indicada como correta, e assim por diante. Quando são ensinadas, por exemplo, as relações AB e BC, com três membros em cada um dos conjuntos A, B e C, participantes humanos geralmente mostram relações emergentes que atestam a formação de três classes de estímulos equivalentes envolvendo os membros relacionados de cada conjunto (A1, B1 e C1 formam uma classe, A2, B2 e C2 formam outra classe e A3, B3 e C3 formam uma terceira classe).

As classes de estímulos equivalentes geradas pelo treino de relações entre estímulos, como em AB e BC, são demonstradas por relações emergentes que atestam as propriedades lógicas da reflexividade, da simetria e da transitividade das relações ensinadas. A demonstração operacional dessas relações emergentes é feita por meio de testes que não empregam qualquer tipo de reforçamento programado ou feedback formal. Testes de identidade podem definir se as relações aprendidas são reflexivas (i.e., se A, então A; se B, então B; se C, então C). Testes de simetria podem ser feitos pela inversão da ordem de uma relação treinada (i.e., se B, então A; se C, então B). A transitividade é determinada pela demonstração de que dois pares de estímulos com um elemento comum estabelecem um novo par (i.e., se A, então C). Simetria e transitividade podem ser demonstradas conjuntamente pelo teste da relação CA (Sidman, 1990; Sidman \& Tailby, 1982). Testes que combinam simetria e transitividade são muitas vezes chamados de testes de equivalência porque evidenciam, simultaneamente, a emergência de duas propriedades das relações de equivalência. A terceira propriedade (reflexiva) é muitas vezes assumida nos estudos com participantes humanos.

Os estudos que adotam o modelo de equivalência têm replicado e confirmado esses dados básicos em uma ampla variedade de condições experimentais, estendendo-os também para questões mais complexas (Sidman, 1994), como a transferência de funções entre estímulos equivalentes. Transferência de funções designa a extensão de efeitos comportamentais de um determinado estímulo para os demais membros da classe (de Rose, McIlvane, Dube, Galpin \& Stoddard, 1988; Dougher, Augustson, Markham, Greenway \& Wulfert, 1994; Hayes, Kohlenberg \& Hayes, 1991; Lyddy, Barnes-Holmes \& Hampson, 2001). Assim, um estímulo que tem determinadas funções pode ser comparado a um "referente" e os estímulos equivalentes a ele podem ser comparados a "símbolos" capazes de substituílos em algumas ocasiões. O método tipicamente empregado para avaliar esse fenômeno envolve, com participantes humanos, o treino e teste de uma classe de equivalência por meio de procedimentos de emparelhamento arbitrário ao modelo. Antes da formação das classes ou seguindo-se a ela, utiliza-se um procedimento experimental para atribuir a um membro dessa classe (em geral um estímulo abstrato) alguma função comportamental. É então testado o surgimento dessa função nos demais membros da classe.

Embora seja um contra-senso matemático admitir variações quantitativas em relações de equivalência, alguns estudos têm mostrado que a transferência de funções entre estímulos equivalentes parece, às vezes, variar em função de parâmetros experimentais. Por exemplo, Fields, LandonJimenez, Buffington e Adams (1995) ensinaram a estudantes universitários relações $\mathrm{AB}, \mathrm{BC}, \mathrm{CD}$ e DE entre figuras abstratas (com dois estímulos em cada conjunto de modelos ou comparações), demonstrando formação de relações de equivalência por meio de testes apropriados. Em seguida, os participantes que estabeleceram classes de equivalência foram treinados a pressionar repetidas vezes uma determinada tecla quando fossem apresentados os estímulos dos conjuntos A e E. Para cada estímulo era necessário um número de pressões diferente. Foi, então, testada a extensão do controle exercido pelos estímulos A1, A2, E1 e E2 para os demais membros de suas respectivas classes. A freqüência com que cada estímulo ocasionava as respostas treinadas para A e E pareceu variar em função do número de nódulos, ou seja, o número de elos intervenientes na relação entre os estímulos, sendo, por exemplo, os estímulos C distantes um nódulo de A, enquanto os estímulos D são distantes dois nódulos de A. Fields et al. sugeriram que a variação no compartilhamento de funções indica que os membros de uma classe de estímulos equivalentes podem ter diferentes "graus de relacionamento" entre si.

O objetivo do presente estudo é apresentar um procedimento desenvolvido para avaliar em que medida ocorre a extensão de funções de "referentes" para "símbolos" em simulações experimentais baseadas no modelo de equivalência de estímulos. Expressões faciais de alegria, raiva e nojo simularam referentes e figuras abstratas simularam símbolos. Expressões faciais foram escolhidas como referentes porque são estímulos considerados naturalmente salientes tanto para humanos quanto para outros primatas na comunicação de sinais sociais (Parr, Winslow, Hopkins \& De Waal, 2000). O reconhecimento de sua relevância talvez se deva ao fato de que a atenção às expressões faciais parece envolvida no processo de evolução que as originou (Öhman, 2002). Isso implica que seríamos hábeis não apenas em produzir sinais comunicativos através da face como também seríamos eficientes em atentar para eles e reagir diferencialmente. Tal hipótese, que já gozava de considerável prestígio advindo talvez de uma extensão dos trabalhos de Ekman e pesquisadores (Ekman, 1972; Ekman, Sorenson \& Friesen, 1969), foi fortalecida por dados psicofisiológicos. Ficou demonstrado que humanos respondem de maneira diferencial e automática com os seus músculos faciais (Dimberg, Thunberg \& Elmehed, 2000), com respostas autonômicas (Esteves, Dimberg \& Öhman, 1994) e com ativação de regiões específicas do cérebro (Morris, Öhman \& Dolan, 1998) quando expostos a faces que expressam condições emocionais. As respostas ocorrem da mesma forma ainda que as faces expressivas 
sejam apresentadas por poucos milissegundos e imediatamente encobertas por faces neutras de modo que não se possa ter "consciência" da apresentação das primeiras (Öhman, 2002). A constatação de que a simples presença de expressões faciais diferentes é condição suficiente para eliciar respostas emocionais distintas torna lícita a suposição de que, por exemplo, faces ameaçadoras e amigáveis tenham naturalmente "significados" diferentes.

O procedimento apresentado neste estudo começa pelo treinamento de relações condicionais para gerar classes de equivalência entre as expressões faciais e as figuras abstratas. Em seguida, os participantes avaliam figuras equivalentes às faces utilizando um conjunto de escalas bipolares ancoradas por adjetivos opostos. Trata-se de um instrumento construído com base na metodologia introduzida por Osgood e pesquisadores (Osgood \& Suci, 1952; Osgood, Suci \& Tannenbaum, 1957) para medir quantitativamente o significado. A metodologia tem o nome de diferencial semântico. O instrumento utilizado neste estudo foi extraído do trabalho de Engelmann (1978), que utilizou o diferencial semântico para medir o significado de relatos verbais de estados subjetivos. A proposta do presente trabalho é que a comparação entre as avaliações das faces (feitas por participantes de um grupo controle) e das figuras equivalentes a elas permita uma medida quantitativa da transferência de funções entre esses estímulos. Dessa forma, será possível avaliar com mais precisão se variações em parâmetros da formação de classes interferem (e em que medida interferem) no grau de relacionamento de estímulos equivalentes.

\section{Método}

\section{Participantes}

Colaboraram com este estudo 40 estudantes recém-admitidos no Curso de Psicologia da Universidade Federal de São Carlos (UFSCar). Esses estudantes foram divididos em dois grupos, sendo o primeiro deles (experimental) composto por 10 participantes e o segundo (controle) composto por 30 participantes.

\section{Situação e Equipamento}

As sessões experimentais a que foram submetidos os participantes do grupo experimental foram conduzidas em uma sala do Laboratório de Estudos do Comportamento Humano (LECH) da Universidade Federal de São Carlos, com razoável isolamento sonoro e de outras interferências externas. Foi utilizado um microcomputador Apple Macintosh Performa 6360 em cuja tela eram apresentados estímulos visuais. O equipamento também registrava as respostas e fornecia as conseqüências. O programa utilizado no experimento foi o software MTS v 10.32 (Dube, 1991). Os estímulos eram apresentados em até cinco "janelas" de $5 \mathrm{X} 5 \mathrm{~cm}$ e os participantes deviam escolhê-los clicando na "janela" correspondente. Na mesma sala desse laboratório, os participantes do grupo experimental preencheram os conjuntos de escalas bipolares que serão mais adiante descritos. Os conjuntos de escalas que couberam aos participantes do grupo controle foram preenchidos em sala de aula.

\section{Procedimento}

Fase 1. Estabelecimento de Equivalência de Estímulos para o Grupo Experimental. A Figura 1 apresenta um diagrama esquemático do treino de discriminações condicionais (emparelhamento com modelo) e do teste de equivalência. $\mathrm{O}$ conjunto A era formado por fotografias de faces humanas, expressando alegria, raiva e nojo, enquanto os conjuntos B, C e D eram formados por três figuras abstratas cada. A Figura 1 mostra uma representação esquemática das relações treinadas e testadas nesta fase.

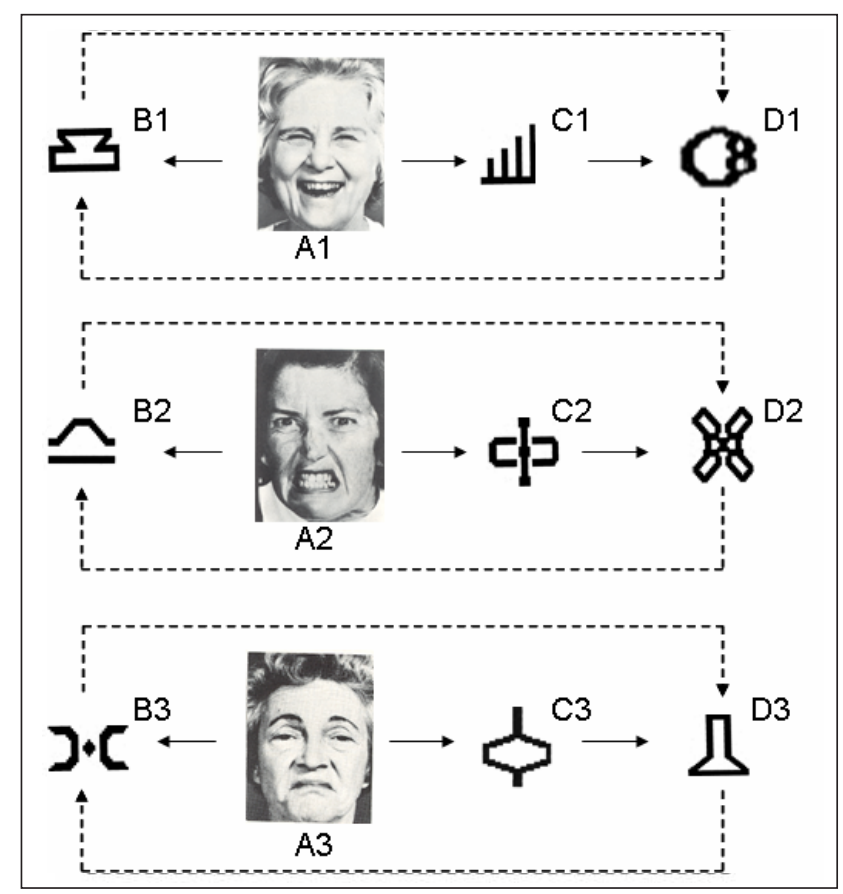

Figura 1. Representação esquemática das relações treinadas (setas contínuas) e testadas (setas tracejadas) na Fase 1.

Inicialmente, foram ensinadas diretamente as relações condicionais $\mathrm{AB}, \mathrm{AC}$ e $\mathrm{CD}$. Cada tentativa de emparelhamento com modelo iniciava-se com a apresentação do estímulo modelo na janela central. Um clique nessa janela, com o mouse, produzia o aparecimento de três estímulos de comparação, em três das janelas periféricas. A outra janela periférica permanecia vazia. Um clique na janela contendo o estímulo considerado correto em presença do modelo apresentado produzia o aparecimento de uma seqüência de tons e um arranjo de estrelas movendo-se na tela do computador. Respostas incorretas produziam o escurecimento da tela por três segundos. (Para uma ilustração desse procedimento de ensino, ver Figura 2). Seguia-se um intervalo entre tentativas de 2 segundos, depois do qual a tentativa seguinte tinha início.

A discriminação condicional $\mathrm{AB}$ foi ensinada em primeiro lugar. $\mathrm{O}$ ensino iniciava-se com um bloco de 36 tentativas AB. Cada tentativa apresentava, portanto, A1, A2 ou A3 como modelo, sendo a seqüência de modelos randomizada ao longo das tentativas, com a restrição de que cada um destes estímulos-modelo era apresentado em um total de 12 tentativas e não ocorria como modelo em mais de 2 tentativas consecutivas. As tentativas $\mathrm{AB}$ apresentavam, como estímulos de comparação, B1, B2 e B3, em três janelas 


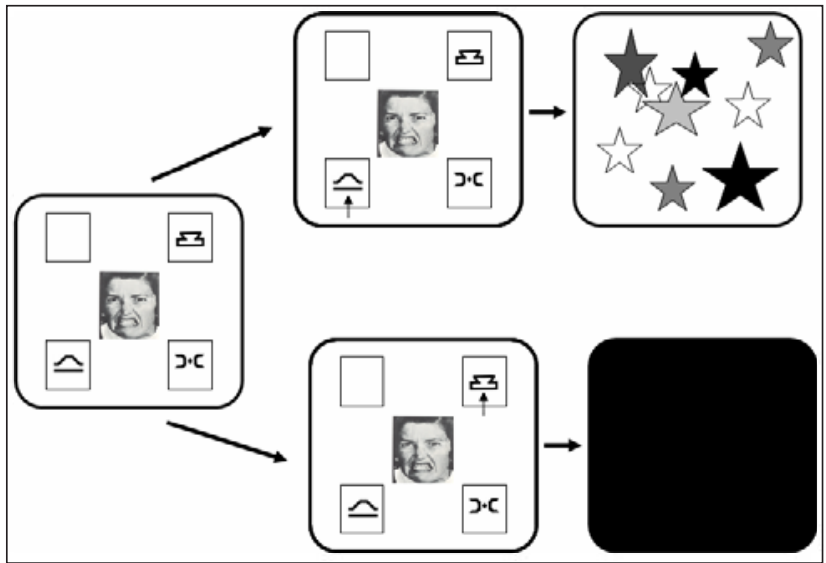

Figura 2. Exemplo de uma tentativa de treino da relação AB. A parte superior da figura mostra uma resposta definida como correta e a conseqüência programada para ela, enquanto a parte inferior mostra uma resposta incorreta também seguida pela sua respectiva conseqüência.

periféricas, ficando a outra janela sem nenhuma figura. A posição de cada figura e da janela vazia (sem figura) mudava de tentativa para tentativa, de acordo com uma seqüência randomizada. Particularmente, a posição da janela que continha a figura designada como correta mudava a cada tentativa, de acordo com uma seqüência randomizada. As primeiras doze tentativas deste bloco apresentavam, além dos estímulos modelo e de comparação, uma instrução escrita, acima do modelo, onde se lia: "Quando esta figura estiver aqui” e acima do estímulo de comparação correto aparecia escrito "escolha esta". Estas instruções deixavam de ser apresentadas a partir da $13^{\text {a }}$ tentativa do bloco. Este bloco de 36 tentativas envolvia, portanto, uma seqüência de 12 tentativas com instrução escrita e 24 tentativas sem instrução. O bloco era repetido até que o participante fizesse escolhas corretas em todas as tentativas. Quando este critério era atingido, considerava-se encerrado o ensino da relação $\mathrm{AB}$ e passava-se ao ensino da relação $\mathrm{AC}$, procedido de maneira análoga. Atingido o critério de escolhas corretas em todas as tentativas, apresentava-se ao participante um bloco que reunia, de maneira intercalada, 12 tentativas do tipo $\mathrm{AB}$ e 12 do tipo AC. Este bloco também era repetido até que as escolhas do participante fossem todas corretas, quando então se passava ao ensino da relação $\mathrm{CD}$ com procedimento semelhante ao usado para ensinar as relações AB e AC.

O participante era submetido, em seguida, a um bloco de 36 tentativas que reunia de maneira intercalada e randômica os três conjuntos de relações ensinadas, AB, AC e CD. Este bloco era repetido até que o participante não apresentasse mais do que um erro. Atingido o critério, aparecia escrito na tela "O computador não vai mais sinalizar se as suas escolhas estão corretas ou erradas" e o bloco era repetido sem o feedback que conseqüenciava as escolhas do participante. Este bloco também era repetido até que não mais do que um erro fosse apresentado. Se o participante errasse em mais do que cinco tentativas, ele era submetido novamente ao bloco anterior (com feedback) antes de repetir o atual.

Para testar a formação de classes de equivalência, eram apresentados dois blocos de 24 tentativas de sonda sem feedback. O primeiro bloco testava se o participante era capaz de estabelecer discriminações condicionais BD, relações que tinham como pré-requisitos a emergência das relações simétricas BA e das relações transitivas AD. Seguia-se, então, o bloco de 36 tentativas sem feedback que reunia de maneira intercalada os três conjuntos de relações ensinadas, AB, AC e CD, repetido sempre que o participante errasse mais do que uma vez. Finalmente, se atingido esse critério, um bloco de 24 tentativas avaliava a emergência de discriminações condicionais DB. Eram pré-requisitos para as relações DB, as relações simétricas DC e CA e as relações transitivas e simétricas DA. Após esse último bloco de sonda, esta fase do experimento era considerada encerrada e o programa fornecia uma mensagem para que o experimentador fosse chamado. Era, então, pedido ao participante que aguardasse em outra sala e, nesse período, o desempenho dele nos blocos de sonda era verificado. O critério para passar à fase seguinte era o de não ter apresentado mais do que um erro em cada um desses blocos de sonda $\mathrm{BD}$ e DB. Caso não atingisse esse critério, o participante não prosseguia no experimento.

Fase 2 - Avaliação dos estímulos através de escalas bipolares. Nesta etapa, cada participante do grupo experimental que atingira o critério de equivalência era instruído a avaliar os estímulos abstratos D1, D2 e D3 de acordo com um conjunto de escalas bipolares. Cada escala se compunha de sete intervalos e era ladeada em suas duas extremidades por "termos polares" constituindo um par de adjetivos antônimos. Vistas em conjunto, representavam uma série de contínuos que iam de um adjetivo ao seu oposto. O conjunto de escalas era impresso em uma folha de papel, que trazia ainda a reprodução de um dos estímulos "D", conforme é exemplificado na Figura 3.

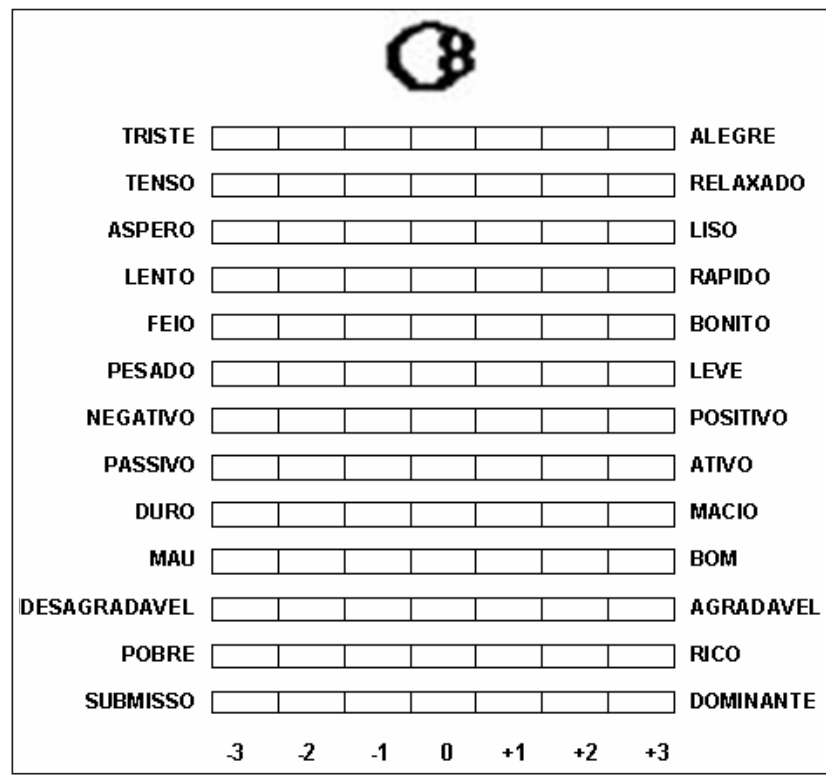

Figura 3. Exemplo de estímulo abstrato e escalas utilizadas pelos participantes nas avaliações dele.

Um único estímulo era reproduzido por lauda, acima do conjunto de escalas. Assim, as avaliações de D1, D2 e D3 foram feitas em folhas separadas, uma para cada estímulo. O participante também recebia uma folha que continha impressas as seguintes instruções:

"Você encontrará desenhos no alto de cada uma das páginas seguintes. Pedimos-lhe que assinale por meio de um X 
o lugar da figura em escalas que são limitadas por dois adjetivos opostos. Cada escala deve ser entendida como um contínuo que vai de um adjetivo ao seu oposto. Assim, você encontrará, por exemplo, o par Bonito/Feio e terá que classificar com relação a este par uma figura qualquer, seja:"

Seguia-se, como exemplo, um desenho abstrato diferente daqueles envolvidos no experimento. Abaixo dele, a instrução escrita continuava da seguinte maneira:

"Se você achar que o desenho acima é extremamente bonito, terá que colocar o X no espaço mais próximo de Bonito. Assim:"

Seguia-se a reprodução da escala BONITO/FEIO com o espaço mais próximo de BONITO assinalado. Abaixo dessa ilustração, continuava a instrução escrita:

"Se achar que é extremamentefeio, terá que colocar o X na outra ponta, no espaço mais próximo de Feio. Assim:"

Seguia-se a reprodução da escala BONITO/FEIO com o espaço mais próximo de FEIO assinalado. Esse padrão era reproduzido para cada um dos outros cinco espaços que poderiam ser assinalados. Depois de fornecer indicações das circunstâncias em que cada espaço deveria ser assinalado, as instruções eram encerradas assim:

"Esta avaliação não é um teste. Não pretende medir inteligência nem caráter e, portanto, não há resposta boa ou má. Procure ser sincero.

Se você tiver alguma dúvida depois de ter lido as instruções, chame o experimentador e faça-lhe perguntas sobre o que não entendeu.

Obrigado pela sua colaboração."

Quando o participante terminava a leitura, o experimentador lhe perguntava se ele havia entendido as instruções. Caso restasse alguma dúvida em relação ao preenchimento das escalas, o experimentador se dispunha a saná-la.
Para o propósito de análise de dados, os espaços receberam valores que variavam de $-3 \mathrm{a}+3$, sendo -3 correspondente à posição mais próxima ao adjetivo negativo e +3 correspondente à posição mais próxima do adjetivo positivo. Para explicitar esses valores, a Figura 3 foi montada com os adjetivos considerados negativos sempre à esquerda e os positivos sempre à direita. Na folha entregue aos participantes, essa disposição era randomizada e os valores que aparecem abaixo das escalas não estavam presentes.

Os participantes do grupo controle receberam as mesmas instruções e foram requisitados a avaliar, além dos estímulos do conjunto D, todas as fotografias de faces expressando emoções. Para o grupo controle, não foi ensinada qualquer relação entre as figuras abstratas e as faces.

\section{Resultados}

Todos os participantes do grupo experimental mostraram desempenhos consistentes na fase de estabelecimento de equivalência de estímulos e puderam avaliar as figuras de acordo com as escalas bipolares. A Figura 4 mostra, em valores absolutos, as médias dos valores atribuídos às avaliações das figuras abstratas D1, D2 e D3 feitas por todos os participantes do grupo experimental em todas as escalas. Da mesma forma, são também mostradas as médias de todas as avaliações das fotografias das expressões faciais de alegria (A1), raiva (A2) e nojo (A3) feitas pelos participantes do grupo controle. A Figura 4 mostra ainda as médias das avaliações que os participantes do grupo controle, não submetidos a qualquer treino de relações entre os estímulos, fizeram das mesmas figuras abstratas avaliadas pelos participantes do grupo experimental (D1, D2 e D3).

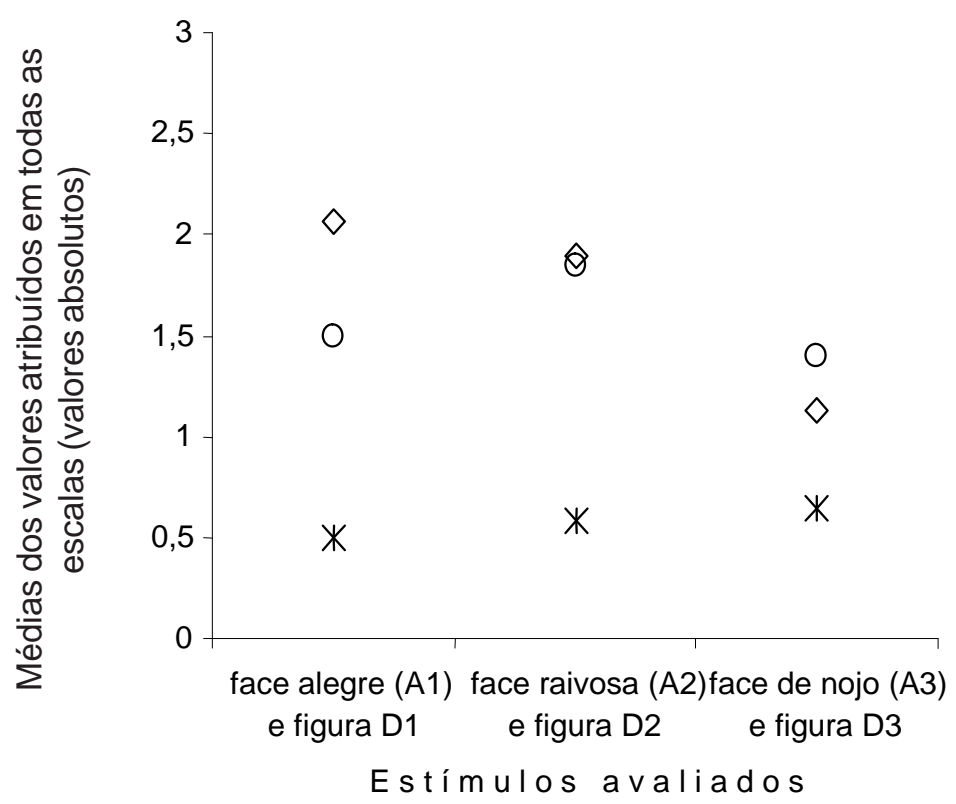

Figura 4. Médias em valores absolutos das avaliações das expressões faciais (feitas pelos participantes do grupo controle) e dos estímulos do conjunto 'D' (grupos experimental e controle). 
Os dados obtidos foram tratados estatisticamente pelo coeficiente de correlação de Kendall, teste que mede a associação entre variáveis mensuradas ao nível ordinal, ou seja, por postos. O teste mostrou significativa correspondência entre as avaliações que os participantes do grupo controle fizeram das expressões faciais de alegria, raiva e nojo e as avaliações dos estímulos D1 (TAUxy = 0.6754, $\mathrm{p}=0.0007$ ), D2 (TAUxy $=0.7186, p=0.0003)$ e D3 $($ TAUxy $=0.6081$, $\mathrm{p}=0.0019)$ feitas pelos participantes do grupo experimental. Particularmente interessante foi que a correspondência verificada entre as avaliações da expressão de raiva e do estímulo abstrato a ela equivalente foi maior do que a correspondência que se encontrou entre as avaliações das expressões de alegria e de nojo com os seus respectivos estímulos equivalentes. Não houve correspondência entre as avaliações das fotografias pelos participantes do grupo controle e as avaliações que eles próprios fizeram dos estímulos D1 (TAUxy= -0.0129, p = 0.5245), D2 (TAUxy = $0.1074, \mathrm{p}=0.6954)$ e D3 (TAUxy $=-0.0774, \mathrm{p}=0.6437)$.

\section{Discussão}

Este estudo apresentou um procedimento para avaliar em que medida "símbolos" adquirem funções de seus "referentes" em simulações experimentais baseadas no modelo de equivalência de estímulos. Trata-se de um procedimento que emprega um método tradicionalmente utilizado na simulação de relações simbólicas combinado com a metodologia introduzida por Osgood e pesquisadores (Osgood \& Suci, 1952; Osgood et al., 1957) para medir quantitativamente o significado. Neste trabalho inicial, estudantes universitários foram submetidos a um treinamento de discriminações condicionais para gerar classes de equivalência entre fotografias de faces expressando emoções e figuras abstratas. Em seguida, eles avaliaram algumas das figuras abstratas utilizando escalas bipolares ancoradas por adjetivos opostos. Um grupo controle, também composto por universitários, avaliou as fotografias e as figuras abstratas utilizando o mesmo conjunto de escalas. As similaridades entre as avaliações das faces pelo grupo controle e das figuras pelo grupo experimental são indicações de que pode haver extensão de "significados" de referentes para símbolos em simulações experimentais estruturadas com base no modelo de equivalência de estímulos. Além de conferir mais validade ao modelo de equivalência, esses resultados indicam que o procedimento apresentado é eficiente na detecção da transferência de funções entre estímulos equivalentes.

O procedimento apresentado neste estudo pode ajudar na avaliação de alguns parâmetros na formação de relações de equivalência, como, por exemplo, a distância nodal entre os estímulos. Nas classes de equivalência geradas a partir das relações ensinadas $\mathrm{AB}$ e $\mathrm{BC}$, os estímulos B são chamados estímulos nodais porque estabelecem as ligações entre os estímulos dos dois outros conjuntos. Nesse caso, diz-se que o conjunto A está separado do conjunto C por um nódulo de distância. Quando são geradas classes de equivalência a partir, por exemplo, das relações treinadas $\mathrm{AB}, \mathrm{BC}$, $\mathrm{CD}$ e DE, três nódulos (B, C e D) mediam a relação entre A e E. A distância nodal entre A e Cé, portanto, menor do que a distância nodal entre A e E. Se parâmetros experimentais da formação de classes, como a distância nodal, implicarem em variações na força das relações estabelecidas entre estímulos equivalentes, o emparelhamento ao modelo não será suficiente para captar esse efeito. Nas simulações experimentais em que o emparelhamento ao modelo é empregado, o participante faz escolhas forçadas a partir das quais se consegue avaliar a formação de classes de equivalência. No entanto, desempenhos consistentes nos testes que avaliam relações emergentes não revelam se os estímulos estão igualmente relacionados entre si. O procedimento apresentado neste estudo parece ser mais efetivo na avaliação de parâmetros experimentais da formação de classes porque combina o emparelhamento ao modelo com uma medida quantitativa de transferência de funções.

Em um estudo preliminar, Caetano, de Rose e Bortoloti (2002a, 2002b) submeteram estudantes universitários a um treinamento de relações condicionais para gerar classes de equivalência envolvendo sete conjuntos de estímulos. Foram ensinadas as relações $\mathrm{AB}, \mathrm{AC}, \mathrm{CD}, \mathrm{DE}, \mathrm{EF}$ e FG, sendo que A era a designação de um conjunto de expressões faciais e B, C, D, E, F e G designações de conjuntos de figuras abstratas. A equivalência foi demonstrada pelas relações GB e BG. Em seguida, um grupo de participantes avaliou os estímulos D (um nódulo distante das faces) e outro grupo avaliou os estímulos $\mathrm{G}$ (quatro nódulos distantes das faces) utilizando o mesmo conjunto de escalas apresentado aqui. Participantes de um grupo controle, não submetidos a qualquer treinamento de relações entre estímulos, avaliaram as faces e as figuras de acordo com as mesmas escalas. As avaliações das faces foram mais similares às avaliações dos estímulos do conjunto $\mathrm{D}$ do que às avaliações dos estímulos do conjunto G. Apesar de ter sido um estudo exploratório, e que ainda necessita de alguns balanceamentos, os resultados obtidos nele indicam que o grau de relacionamento entre as faces e os estímulos abstratos decresceu em função do número de nódulos, apoiando as conclusões de Fields, Adams, Verhave, e Newman (1990) e Fields et al. (1995). Além disso, o procedimento permitiu quantificar a magnitude do enfraquecimento das relações ao longo da cadeia nodal.

Caetano, de Rose e Bortoloti (2002a, 2002b) verificaram diferenças no grau de relacionamento entre membros de uma mesma classe, mas esse procedimento também permite comparar o nível de transferência de funções entre classes diferentes. Podem ser manipulados e avaliados outros parâmetros da formação de classes como o "atraso" na apresentação dos estímulos de comparação em tarefas de emparelhamento ao modelo. Nesse caso, é possível pensar no envolvimento de dois grupos de participantes que seriam ensinados a estabelecer as mesmas relações $\mathrm{AB}, \mathrm{AC}$ e $\mathrm{CD}$, sendo a equivalência testada pela emergência de DB e BD. Para um dos grupos, modelo e comparações apareceriam simultaneamente, na mesma tela; para o outro grupo, o modelo apareceria primeiro, seria removido e, após um intervalo determinado, apareceriam os estímulos de comparação. Para o segundo grupo, portanto, modelo e comparações nunca ficariam presentes na 
mesma tela e haveria um atraso na apresentação dos estímulos de comparação. O conjunto A também seria composto por estímulos significativos, como expressões faciais, e os conjuntos B, C e D seriam compostos por figuras abstratas. Os participantes desses dois grupos avaliariam os estímulos D e essas avaliações seriam comparadas com as avaliações dos estímulos A, feitas por participantes de um grupo controle. Dessa forma, seria possível avaliar se o grau de relacionamento de estímulos equivalentes é afetado pela apresentação atrasada dos estímulos de comparação e em que medida ele é afetado. Também é possível pensar em um delineamento experimental combinando a apresentação atrasada dos estímulos de comparação com a distância nodal para avaliar a influência de um parâmetro sobre o outro.

Este estudo permite ainda uma especulação derivada do fato de ter havido mais similaridade entre as avaliações da face raivosa e seu símbolo do que entre as avaliações da face alegre e seu símbolo. Expressões faciais ameaçadoras "avisam" que conseqüências aversivas são possíveis e por isso seriam capazes de capturar mais da nossa atenção do que expressões amigáveis. Haveria uma vantagem evolutiva no rápido reconhecimento de um potencial agressor. Muitos trabalhos têm mostrado de forma consistente que expressões ameaçadoras em multidões amigáveis são melhor e mais rapidamente detectadas do que faces amigáveis em multidões ameaçadoras (Esteves, 1999; Hansen \& Hansen, 1988; Mogg \& Bradley, 1999; Öhman, Lundqvist \& Esteves, 2001; White, 1996). Os resultados deste estudo dão margem à especulação de que a maior eficiência de algumas respostas humanas diante de uma expressão facial de raiva talvez se possa estender para os estímulos equivalentes a ela. Assim, se essa extensão de funções de fato ocorreu, ela pode ter contribuído para uma similaridade maior na avaliação que se fez da face raivosa e da figura abstrata equivalente a ela. Essa hipótese necessita, contudo, de uma confirmação empírica mais consistente.

\section{Referências}

Caetano, M. S., de Rose, J. C., \& Bortoloti, R. (2002a). Relatedness of equivalent stimuli as a function of the number of nodes. Trabalho apresentado no 28th Annual Convention of Association for Behavior Analysis, Toronto, Canadá.

Caetano, M. S., de Rose, J. C., \& Bortoloti, R. (2002b). Medindo o grau de equivalência de estímulos em função do número de nódulos. [Resumo]. In Sociedade Brasileira de Psicologia (Ed.), Resumos de comunicações científicas, XXXII Reunião Anual de Psicologia. Ribeirão Preto, SP: SBP.

de Rose, J. C., Mcilvane, W. J., Dube, W. V., Galpin, V. C., \& Stoddard, L. T. (1988). Emergent simple discriminations established by indirect relations to differential consequences. Journal of the Experimental Analysis of Behavior, 50, 1-20.

Dimberg, U., Thunberg, M., \& Elmehed, K. (2000). Unconscious facial reactions to emotional facial expressions. Psychological Science, 11, 86-89.

Dougher, M., Augustson, E., Markham, M., Greenway, D., \& Wulfert, E. (1994). The transfer of respondent eliciting and extinction functions through stimulus equivalence classes. Journal of the Experimental Analysis of Behavior, 62, 331-351.

Dube, W. V. (1991). Computer software for stimulus control research with Macintosh computers. Experimental Analysis of Human Behavior Bulletin, 9, 28-30.
Ekman, P. (1972). Darwin and facial expression: A century of research in review. New York: Academic Press.

Ekman, P., Sorenson, E. R., \& Friesen, W. V. (1969). Pan-cultural elements in facial displays of emotion. Science, 164, 86-88.

Engelmann, A. (1978). Os estados subjetivos, uma tentativa de classificação de seus relatos verbais. São Paulo, SP: Ática.

Esteves, F. (1999). Attentional bias to emotional facial expressions. European Review of Applied Psychology, 49(2), 91-96.

Esteves, F., Dimberg, U., \& Öhman, A. (1994). Automatically elicited fear: Conditioned skin conductance responses to masked facial expressions. Cognition and Emotion, 8, 383-413.

Fields, L., Adams, B. J., Verhave, T., \& Newman, S. (1990). The effects of nodality on the formation of equivalence classes. Journal of the Experimental Analysis of Behavior, 53, 345-358.

Fields, L., Landon-Jimenez, D. V., Buffington, D. M., \& Adams, B. J. (1995). Maintained nodal-distance effects in equivalence classes. Journal of the Experimental Analysis of Behavior, 64, 129-145.

Hansen, C., \& Hansen, R. (1988). Finding the face in the crowd: An anger superiority effect. Journal of Personality and Social Psychology, 54, 917-24.

Hayes, S. C., Kohlenberg, B. S., \& Hayes, L. J. (1991). The transfer of contextual control over equivalence classes through equivalence classes: A possible model of social stereotyping. Journal of the Experimental Analysis of Behavior, 56, 505-518.

Lyddy, F., Barnes-Holmes, D., \& Hampson, P. J. (2001). A transfer of sequence function via equivalence in a connectionist network. The Psychological Record, 51, 409-428.

Mogg, K., \& Bradley, B. P. (1999). Orienting of attention to threatening facial expressions presented under conditions of restricted awareness. Cognition and Emotion, 13, 713-740.

Morris, J.S., Öhman, A., \& Dolan, R. J. (1998). Conscious and unconscious emotional learning in the amygdala. Nature, 393, 467-470.

Öhman, A. (2002). Automaticity and the amygdala: Nonconscious responses to emotional faces. Current Directions in Psychological Science, 11(2), 62-66.

Öhman, A., Lundqvist, D., \& Esteves, F. (2001). The face in the crowd revisited: A threat advantage with schematic stimuli. Journal of Personality and Social Psychology, 80, 381-396.

Osgood, C. E., \& Suci, G. I. (1952). A measure of relation determined by both mean difference and profile information. Psychological Bulletin, 49, 251-262.

Osgood, C. E., Suci, G. I., \& Tannenbaum, P. H. (1957). The measurement of meaning. Urbana, IL: University of Illinois Press.

Parr, L. A., Winslow, J. T., Hopkins, W. D., \& De Waal, F. B. M. (2000). Recognizing facial cues: Individual discrimination by chimpanzees (Pan troglodytes) and rhesus monkeys (Macaca mulatta). Journal of Comparative Psychology, 114, 47-60.

Sidman, M. (1990). Equivalence relations: Where do they come from? In H. Lejeune \& D. Blackman (Eds.), Behavior analysis in theory and practice: Contributions and controversies (pp. 93-114). Hillsdale, NJ: Erlbaum.

Sidman, M. (1994). Equivalence relations and Behavior: A research story. Boston: Authors Cooperative.

Sidman, M., \& Tailby, W. (1982). Conditional discrimination vs. matching-to-sample: An expansion of the testing paradigm. Journal of the Experimental Analysis of Behavior, 37, 261-273.

White, M (1996). Anger recognition is independent of spatial attention. New Zealand Journal of Psychology, 21(1), 30-35.

Recebido: 02/09/2005 $1^{a}$ revisão: 06/06/2006 $2^{a}$ revisão: $20 / 10 / 2006$ Aceite final: 23/10/2006 